\title{
Definitive Radiotherapy versus Postoperative Radiotherapy of Patients with Oro- and Hypopharyngeal Cancer: Impact of Prognostic Factors
}

\author{
Volker Rudat, ${ }^{1,2}$ Salia Ahmet-Osman, ${ }^{1}$ Oliver Schramm, ${ }^{1}$ and Andreas Dietz \\ ${ }^{1}$ Department of Radiation Oncology, University of Heidelberg, Heidelberg 69120, Germany \\ ${ }^{2}$ Department of Radiation Oncology, Saad Specialist Hospital, Al-Khobar 31952, Saudi Arabia \\ ${ }^{3}$ Department of Head and Neck Surgery, University of Leipzig, Leipzig 04103, Germany
}

Correspondence should be addressed to Volker Rudat, volker.rudat@gmail.com

Received 11 September 2011; Accepted 9 October 2011

Academic Editor: Dirk Rades

Copyright ( 2012 Volker Rudat et al. This is an open access article distributed under the Creative Commons Attribution License, which permits unrestricted use, distribution, and reproduction in any medium, provided the original work is properly cited.

\begin{abstract}
Purpose. To compare the impact of prognostic factors of patients treated with definitive radio(chemo)therapy versus patients treated with surgery and postoperative radiotherapy for squamous cell carcinoma of the oro- and hypopharynx. Patients and Methods. 162 patients treated with definitive radiotherapy and 126 patients treated with postoperative radiotherapy were retrospectively analysed. The impact of the prognostic factors gender, age, total tumor volume (TTV), pre-radiotherapy hemoglobin level (Hb-level), tumor site, $\mathrm{T}$ - and $\mathrm{N}$-classification, radiotherapy interruptions $>5$ days, radiotherapy versus simultaneous radiochemotherapy, R-status and time interval between surgery and radiotherapy were investigated. Results. The median follow-up time for the censored patients treated with definitive radio(chemo)therapy was 28.5 months and for postoperative radiotherapy 36.5 months. On univariate analysis, the TTV, Hb-level, and simultaneous radiochemotherapy had a significant impact on the survival of patients treated with definitive radio(chemo)therapy. For patients treated with postoperative radiotherapy, only the TTV showed a statistical trend for the survival $(P=0.13)$. On multivariate analysis, the TTV and simultaneous radiochemotherapy maintained their statistical significance for patients treated with definitive raditherapy, and the TTV, the statistical trend for patients treated with postoperative radiotherapy $(P=0.19)$. Conclusions. The TTV was the predominant prognostic factor for both, patients treated with definitive or postoperative radiotherapy.
\end{abstract}

\section{Introduction}

Squamous cell carcinoma of the head and neck (SCCHN) is the fifth most common neoplasm with an estimated annual global incidence of more than 500,000 cases diagnosed worldwide [1]. The treatment is usually interdisciplinary and mainly involves surgeons, radiation oncologists, medical oncologists, clinical nurse specialists, speech and language specialists, and dieticians [2]. Dependent on the situation, goals of the treatment can be to obtain (i) a high locoregional control and survival rates in patients with limited disease, (ii) an increased survival in patients with advanced disease (improved locoregional control, reduced probability of distant metastasis, and second malignancies), (iii) an increased organ-function preservation in resectable and unresectable tumors, and (vi) an increased therapeutic ratio (cure/toxicity ratio) [3]. Single modality treatment is recommended for the patients with early-stage disease (stage I or stage II, approximately $40 \%$ of the patients with SCCHN) and combined modality treatment for patients with locally advanced disease. The combined modality treatment may include surgery followed by adjuvant radiotherapy or radiochemotherapy, concomitant radiochemotherapy (using conventional or alternative fractionation regimen), and induction chemotherapy followed by radiotherapy or radiochemotherapy $[3,4]$.

A precise understanding of prognostic factors is important to select the optimal treatment for the individual patient or to stratify patients for clinical trials or statistical analyses. In this retrospective single-institutional study, the role of potential prognostic factors was evaluated and compared in patients with squamous cell carcinoma of 
the oro- and hypopharynx after treatment with definitive radiotherapy/radiochemotherapy (dRT) versus surgery followed by postoperative radiotherapy (pRT).

\section{Patients and Methods}

Between 1992 and 2000, 288 patients with squamous cell carcinoma of the oropharynx or hypopharynx received a radiation therapy as definitive $(n=162)$ or as postoperative $(n=126)$ treatment.

Eligibility criteria for this retrospective single-institutional study were histologically proven squamous cell carcinoma of the oropharynx or hypopharynx, no distant metastasis or synchronous cancer at time of diagnosis, and definitive or postoperative radiotherapy with a minimum total dose of $60 \mathrm{~Gy}$.

\subsection{Radiation Therapy and Simultaneous Chemotherapy.} One hundred and thirty-eight of $162(85 \%)$ patients treated with definitive radiotherapy received a concomitant boost fractionation regimen, and $24(15 \%)$ were treated with conventional fractionation (single fraction dose of $2 \mathrm{~Gy}$, one fraction a day, five fractions a week). Two concomitant boost regimen were used: Regimen 1 consisted of a total dose of $66 \mathrm{~Gy}$ in five weeks with a daily fraction dose of $2 \mathrm{~Gy}$ and a concomitant boost of $1.6 \mathrm{~Gy}$ during the last two weeks $(n=76)$ and regimen 2 of a total dose of 69.9 Gy in 5.5 weeks with a daily fraction dose of $1.8 \mathrm{~Gy}$ and a concomitant boost of 1.5 Gy during the last 2.5 weeks $(n=62)$.

Ninety-four of 138 (68\%) patients treated with concomitant boost fractionation regimen received a simultaneous chemotherapy as well as five (4\%) patients treated with conventional fractionation.

The simultaneous chemotherapy consisted of $70 \mathrm{mg} / \mathrm{m}^{2}$ Carboplatin on days $1-5$ and $29-33(n=73)$ or $70 \mathrm{mg} / \mathrm{m}^{2}$ Carboplatin and $600 \mathrm{mg} / \mathrm{m}^{2}$ 5-fluorouracil on days $1-5$ and 29-33 $(n=26)$. Carboplatin was administered as a daily short-term intravenous infusion and 5-fluorouracil as an intravenous continuous infusion for 120 hours.

For the postoperative radiotherapy, only conventional fractionation without simultaneous chemotherapy was used $(n=123)$.

The radiotherapy was performed with opposed lateral fields for the upper neck and one anterior field for the lower neck using $6 \mathrm{MeV}$ photons. Patients were treated in a thermoplastic mask for immobilization, and individual blocks were used to spare normal tissue where possible. After a dose of 30 to $36 \mathrm{~Gy}$ to the reference point, the spinal cord was spared out of the photon fields and the uninvolved posterior neck treated with electrons of selected energy according to CT findings with daily doses of $2.5 \mathrm{~Gy}$ five times a week to the prescribed total dose. Target volumes were defined on CT scans, and the dose was calculated to midplane. In selected patients, three-dimensional treatment planning was performed and conformal treatment techniques used.

2.2. Quantitative Determination of the Total Tumor Volume (TTV) from Digitised CT Scans. Pre-treatment CT scans of all patients were digitized with an automatic laser scanner (FIPS PLUS). The stored images were transferred to a personal computer. The macroscopic tumor shape (primary tumor and locoregional lymph node metastases) was defined in every CT slice (slice thickness $5 \mathrm{~mm}$ or $8 \mathrm{~mm}$ ) using a drawing tool (software: photostyler). The number of pixels $n$ enclosed by this contour was determined with a customshaped image processing program (software: Interactive Data Language). The area $A_{i}$ of the $i$ th slice was determined as $A_{i}=$ pixel size (length) $*$ pixel size (width) $* n$. The pixel size was determined using the scaling as given on the CT-hardcopy. The determined tumor area of each slice was multiplied with its slice thickness $d_{i}$. The TTV was approximated by

$$
V_{\text {tumor }}\left(\mathrm{cm}^{3}\right)=\sum_{i-1}^{m} A_{i}\left(\mathrm{~mm}^{2}\right) \times d_{i}(\mathrm{~mm}) / 1000 .
$$

No interpolation between the CT slices was performed.

Repeated measurements using irregularly shaped tumor phantoms showed a difference between the reference volumes and CT-based volume measurements depending of the slice thickness ( $5 \mathrm{~mm}$ or $8 \mathrm{~mm}$ ) of $1.4 \%$ to $4.5 \%$.

\subsection{Estimation of the Total Tumor Volume (TTV) Based on the Postoperative Histopathological Report. In addition to the quantitative tumor volumetry based on digitised pre- treatment CT scans, TTV was estimated based on the postoperative histopathological report in patients treated with adjuvant radiotherapy. The TTV was approximated using the equation $V=4 / 3 \pi * a * b * c$, where $a, b$, and $c$ represent the orthogonal maximal tumor diameters.}

2.4. Statistical Analysis. The overall survival was defined as the time between the first day of the treatment and death of any cause. The overall survival was estimated using the Kaplan-Meier method, and treatment groups were compared using a two-sided log rank test. The locoregional failure-free survival was defined as the time between the first day of the treatment and a locoregional failure. The distant metastasisfree survival was defined as the time between the first day of the treatment and a distant failure. The locoregional failure-free survival and the distant metastasis-free survival were estimated by the cumulative incidence method, and treatment groups were compared using the Gray test $[5,6]$.

The simultaneous relationship of multiple prognostic factors to overall survival was assessed using Cox's proportional hazard regression analysis. The simultaneous relationship of multiple prognostic factors to locoregional failurefree or distant metastasis-free survival was assessed using the hazards of the cumulative incidence function model [7].

To estimate the reliability of the tumor volumetry, the TTV derived from digitised pretreatment CT scans was compared with the TTV derived from calculations based on tumor diameters provided by the postoperative histopathological report of the same patients. For the method comparison, the limits of agreement were estimated as described by Bland and Altman [8]. 


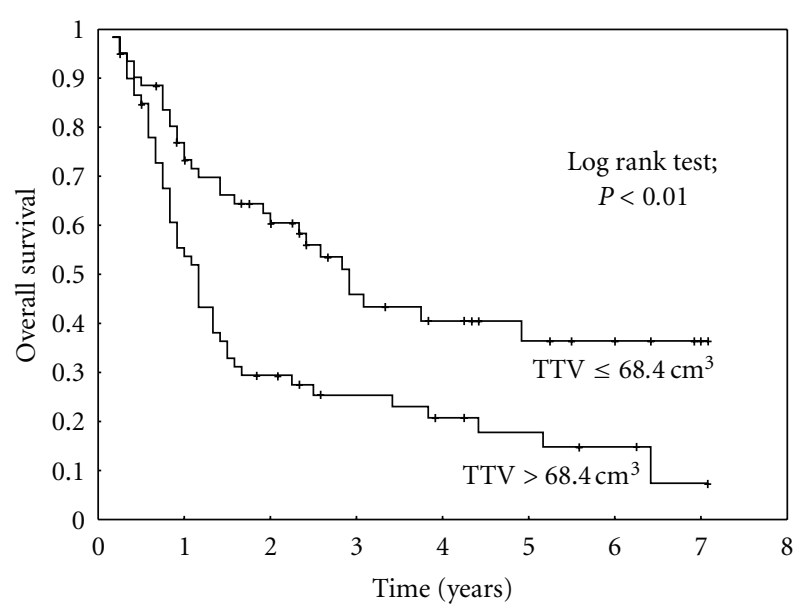

Figure 1: Impact of the total tumor volume (TTV) on overall survival in patients treated with definitive radiotherapy/radiochemotherapy.

\section{Results}

The median follow-up time for the censored patients treated with definitive radiotherapy/radiochemotherapy was 28.5 months and for the patients treated with adjuvant postoperative radiotherapy 36.5 months. The 5 -year overall survival for the patients treated with definitive radiotherapy/radiochemotherapy was 0.27 (95\% CI $0.18-0.35)$ and for the patients treated with postoperative radiotherapy, 0.69 (95\% CI 0.59-0.79).

The patient and treatment characteristics are demonstrated in Table 1. Patients treated with definitive radiotherapy/radiochemotherapy had a greater proportion of stage 4 tumors (96.9\% versus 66.9\%), a much larger median TTV $\left(68.4 \mathrm{~cm}^{3}\right.$ versus $\left.21.2 \mathrm{~cm}^{3}\right)$, and a lower proportion of preradiotherapy hemoglobin level $\leq 12 \mathrm{~g} / \mathrm{dL}$ (17.6\% versus $42.6 \%$ ). The reduced $\mathrm{Hb}$ level was probably due to surgeryrelated blood loss. The performance status was not considered in this study because it was not consistently documented in the patient files.

3.1. Definitive Radiotherapy/Radiochemotherapy. On univariate analysis, the TTV (Figures 1 and 2) and the preradiotherapy hemoglobin level had a statistically significant impact on the overall survival and on the locoregional control of patients treated with definitive radiotherapy/ radiochemotherapy. Simultaneous chemotherapy had a statistically significant effect on the overall survival and on the incidence of distant metastasis. In addition, there was a statistical trend of an association of simultaneous chemotherapy with the locoregional control $(P=0.08)$. The $\mathrm{N}$-classification had a statistically significant impact on locoregional control but no significant impact on the overall survival or metastasis-free survival. The results of the univariate analysis are summarized in Table 2. On multivariate analysis, only the TTV and the simultaneous chemotherapy maintained their statistical significance (Table 3).

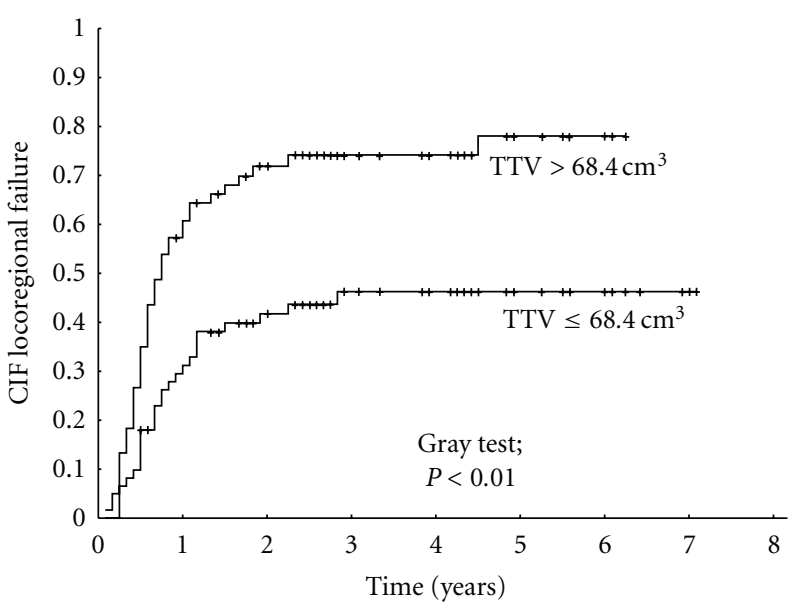

FIGURE 2: Impact of the total tumor volume (TTV) on locoregional control in patients with definitive radiotherapy/radiochemotherapy. CIF: cumulative incidence function.

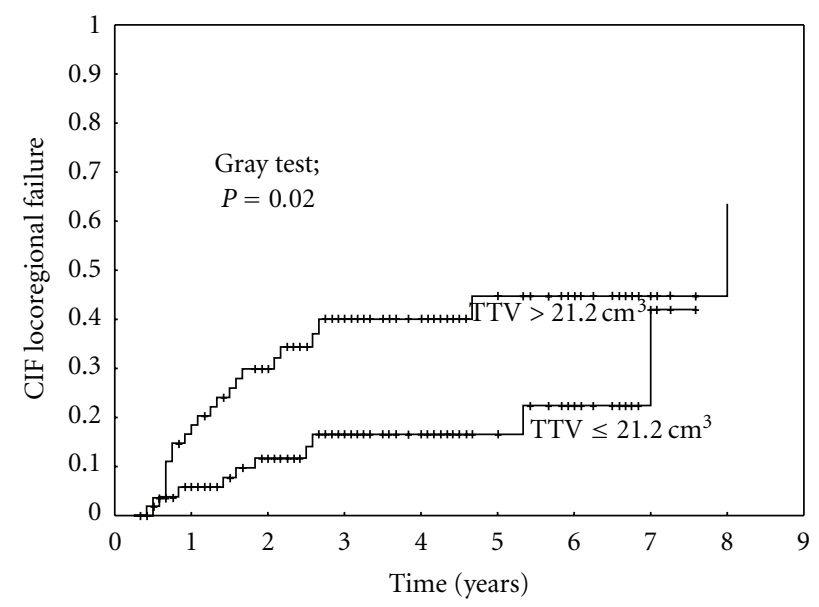

FIGURE 3: Impact of the total tumor volume (TTV) on locoregional control in patients treated with postoperative radiotherapy. CIF: cumulative incidence function.

3.2. Postoperative Radiotherapy. On univariate analysis, the TTV had a statistically significant impact on the locoregional control (Figure 3). The N-classification had a statistically significant impact on the incidence of distant metastasis and a statistical trend on the locoregional control $(P=0.06)$. The results of the univariate analysis are summarized in Table 4. On multivariate analysis, only the TTV $(P=0.05)$ maintained its statistical significance (Table 5).

3.3. Tumor Volumetry Method Comparison. The TTV was estimated using quantitative tumor volumetry of digitized pre-treatment CT scans in all patients. In addition to the CT-based volumetry, in 34 patients, the TTV was also estimated based on tumor diameters reported in the histopathological report. The tumor volumes based on the two methods were compared to estimate the precision of the 
TABLE 1: Patient characteristics.

\begin{tabular}{|c|c|c|c|c|}
\hline \multirow{2}{*}{ Characteristics } & \multicolumn{2}{|c|}{ Definitive RT } & \multicolumn{2}{|c|}{ Postoperative RT } \\
\hline & $n$ & $\%$ & $n$ & $\%$ \\
\hline \multicolumn{5}{|l|}{ Gender } \\
\hline Female & 17 & 10.5 & 19 & 15.1 \\
\hline Male & 145 & 89.5 & 107 & 84.9 \\
\hline \multicolumn{5}{|l|}{ Age (years) } \\
\hline$\leq 56$ & 87 & 53.7 & 72 & 57.1 \\
\hline$>56$ & 75 & 46.3 & 54 & 42.9 \\
\hline \multicolumn{5}{|c|}{ Hemoglobin level pre-RT (g/dL) } \\
\hline$\leq 12$ & 27 & 17.6 & 46 & 42.6 \\
\hline$>12$ & 126 & 82.4 & 62 & 57.4 \\
\hline \multicolumn{5}{|l|}{ Tumor site } \\
\hline Oropharynx & 95 & 58.6 & 68 & 54.0 \\
\hline Hypopharynx & 67 & 41.4 & 58 & 46.0 \\
\hline \multicolumn{5}{|l|}{ cT-Classification ${ }^{\#}$} \\
\hline 1,2 & 13 & 8.1 & 66 & 52.4 \\
\hline 3,4 & 148 & 91.9 & 60 & 47.6 \\
\hline \multicolumn{5}{|l|}{$\mathrm{cN}^{-C l a s s i f i c a t i o n}{ }^{\#}$} \\
\hline 0,1 & 14 & 3.7 & 50 & 39.7 \\
\hline 2,3 & 148 & 91.3 & 76 & 60.3 \\
\hline \multicolumn{5}{|l|}{ Stage } \\
\hline$\leq 3$ & 4 & 3.1 & 42 & 33.3 \\
\hline 4 & 157 & 96.9 & 84 & 66.7 \\
\hline \multicolumn{5}{|l|}{ Total tumor volume } \\
\hline$\leq$ median* & 52 & 43.0 & 52 & 46.8 \\
\hline$>$ median* & 69 & 57.0 & 59 & 53.2 \\
\hline \multicolumn{5}{|l|}{ R-Classification } \\
\hline 0 & - & - & 78 & 65.0 \\
\hline 1 & - & - & 37 & 30.8 \\
\hline 2 & - & - & 5 & 4.2 \\
\hline \multicolumn{5}{|l|}{ Fractionation regimen } \\
\hline Conventional & 24 & 14.8 & 126 & 100.0 \\
\hline Concomitant boost & 138 & 85.2 & 0 & 0.0 \\
\hline \multicolumn{5}{|c|}{ Simultaneous radiochemotherapy } \\
\hline Yes & 99 & 61.1 & 0 & 0.0 \\
\hline No & 63 & 38.9 & 126 & 100.0 \\
\hline \multicolumn{5}{|l|}{ RT interruptions $>5$ days } \\
\hline Yes & 36 & 22.2 & 24 & 20.0 \\
\hline No & 126 & 77.8 & 96 & 80.0 \\
\hline \multicolumn{5}{|c|}{ Interval surgery-RT $>32$ days } \\
\hline Yes & - & - & 58 & 47.5 \\
\hline No & - & - & 64 & 52.5 \\
\hline
\end{tabular}

$\mathrm{RT}$, radiation therapy; * median for definitive RT is $69.4 \mathrm{~cm}^{3}$, median for postoperative RT is $21.2 \mathrm{~cm}^{3}$.

tumor volume measurements. The method comparison of the 34 patients showed that the $95 \%$ limit of agreement between the two total tumor volume measurements was approximately $\pm 150 \%$ of the average total tumor volume measurement of both methods (Figure 4).

\section{Discussion}

This retrospective single institution analysis investigated possible prognostic factors of patients with squamous cell cancer of the oro- and hypopharynx treated with (i) definitive 
TABLE 2: Definitive radiation therapy: univariate analysis of overall survival, locoregional control, and metastasis-free survival.

\begin{tabular}{|c|c|c|c|c|c|c|}
\hline \multirow{2}{*}{ Factor } & \multicolumn{2}{|l|}{ Overall survival } & \multicolumn{2}{|l|}{ Locoregional failure } & \multicolumn{2}{|l|}{ Metastasis } \\
\hline & $5 y$-survival & $P$ & $5 y-C I F$ & $P$ & $5 y-C I F$ & $P$ \\
\hline \multicolumn{7}{|l|}{ Gender } \\
\hline Female & 0.15 (95\% CI $0.01-0.45)$ & & 0.60 (95\% CI $0.50-0.68)$ & & $0.22(95 \%$ CI $0.04-0.49)$ & \\
\hline Male & 0.28 (95\% CI 0.19-0.37) & 0.39 & $0.61(95 \%$ CI $0.52-0.70)$ & 0.52 & 0.33 (95\% CI $0.24-0.42)$ & 0.47 \\
\hline \multicolumn{7}{|l|}{ Age (years) } \\
\hline$\leq 56$ & 0.27 (95\% CI 0.17-0.39) & & $0.63(95 \%$ CI $0.51-0.73)$ & & $0.34(95 \%$ CI $0.23-0.45)$ & \\
\hline$>56$ & $0.25(95 \%$ CI $0.13-0.40)$ & 0.43 & $0.63(95 \%$ CI $0.46-0.76)$ & 0.29 & $0.28(95 \%$ CI $0.17-0.40)$ & 0.61 \\
\hline \multicolumn{7}{|c|}{$\begin{array}{l}\text { Hemoglobin level pre-RT } \\
(\mathrm{g} / \mathrm{dL})\end{array}$} \\
\hline$\leq 12$ & $0.09(95 \%$ CI $0.01-0.30)$ & & $0.82(95 \%$ CI $0.56-0.93)$ & & 0.25 (95\% CI 0.07-0.48) & \\
\hline$>12$ & $0.30(95 \%$ CI $0.20-0.40)$ & 0.02 & $0.58(95 \%$ CI $0.47-0.67)$ & 0.01 & $0.33(95 \%$ CI $0.23-0.42)$ & 0.46 \\
\hline \multicolumn{7}{|l|}{ Tumor site } \\
\hline Oropharynx & $0.24(95 \%$ CI $0.14-0.35)$ & & $0.69(95 \%$ CI $0.56-0.78)$ & & $0.34(95 \%$ CI $0.24-0.44)$ & \\
\hline Hypopharynx & $0.30(95 \%$ CI $0.17-0.44)$ & 0.70 & $0.54(95 \%$ CI $0.39-0.67)$ & 0.14 & $0.28(95 \%$ CI $0.16-0.42)$ & 0.32 \\
\hline \multicolumn{7}{|l|}{ T-Classification } \\
\hline 1,2 & 0.44 (95\% CI 0.13-0.72) & & 0.41 (95\% CI 0.13-0.67) & & $0.25(95 \%$ CI $0.05-0.51)$ & \\
\hline 3,4 & 0.25 (95\% CI 0.17-0.34) & 0.34 & 0.65 (95\% CI $0.55-0.73)$ & 0.28 & 0.33 (95\% CI $0.24-0.42)$ & 0.70 \\
\hline \multicolumn{7}{|l|}{ N-Classification } \\
\hline 0,1 & 0.52 (95\% CI 0.19-0.77) & & 0.17 (95\% CI 0.02-0.43) & & $0.16(95 \%$ CI $0.02-0.41)$ & \\
\hline 2,3 & $0.26(95 \%$ CI $0.17-0.34)$ & 0.17 & $0.66(95 \%$ CI $0.56-0.74)$ & $<0.01$ & $0.33(95 \%$ CI $0.24-0.41)$ & 0.38 \\
\hline \multicolumn{7}{|c|}{ Total tumor volume $\left(\mathrm{cm}^{3}\right)$} \\
\hline$\leq 68.4$ & $0.36(95 \%$ CI $0.22-0.51)$ & & $0.46(95 \%$ CI $0.33-0.59)$ & & $0.29(95 \%$ CI $0.15-0.44)$ & \\
\hline$>68.4$ & 0.18 (95\% CI 0.09-0.30) & $<0.01$ & 0.78 (95\% CI 0.62-0.88) & $<0.01$ & 0.37 (95\% CI $0.24-0.50)$ & 0.13 \\
\hline \multicolumn{7}{|c|}{$\begin{array}{l}\text { Simultaneous } \\
\text { radiochemotherapy }\end{array}$} \\
\hline Yes & 0.32 (95\% CI 0.21-0.42) & & 0.57 (95\% CI 0.46-0.67) & & 0.37 (95\% CI 0.27-0.48) & \\
\hline No & 0.17 (95\% CI $0.05-0.37)$ & 0.03 & $0.73(95 \%$ CI $0.53-0.85)$ & 0.08 & $0.21(95 \%$ CI $0.1-0.34)$ & 0.04 \\
\hline \multicolumn{7}{|c|}{ RT interruptions $>5$ days } \\
\hline Yes & 0.15 (95\% CI $0.01-0.43)$ & & 0.58 (95\% CI 0.29-0.78) & & $0.28(95 \%$ CI $0.13-0.44)$ & \\
\hline No & 0.28 (95\% CI $0.19-0.38)$ & 0.51 & $0.64(95 \%$ CI $0.53-0.73)$ & 0.83 & $0.33(95 \%$ CI $0.23-0.42)$ & 0.96 \\
\hline
\end{tabular}

RT, radiation therapy; CIF, cumulative incidence function; $P$ : $P$ value.

TABLE 3: Definitive radiation therapy: multivariate analysis of overall survival, locoregional control, and metastasis-free survival.

\begin{tabular}{|c|c|c|c|c|c|c|}
\hline \multirow{2}{*}{ Factor } & \multicolumn{2}{|l|}{ Overall survival } & \multicolumn{2}{|c|}{ Locoregional control } & \multicolumn{2}{|c|}{ Metastasis-free survival } \\
\hline & $\mathrm{RR}$ & $P$ & $\mathrm{RR}$ & $P$ & $\mathrm{RR}$ & $P$ \\
\hline Gender & $1.34(95 \%$ CI $0.62-2.91)$ & 0.46 & $1.50(95 \%$ CI $0.70-3.24)$ & 0.30 & 0.79 (95\% CI 0.15-4.07) & 0.78 \\
\hline Age & $1(95 \%$ CI $0.63-1.59)$ & 0.99 & 1.20 (95\% CI 0.74-1.95) & 0.45 & 0.96 (95\% CI 0.47-1.98) & 0.91 \\
\hline Hemoglobin level pre-RT & 0.74 (95\% CI $0.41-1.35)$ & 0.33 & $0.74(95 \%$ CI $0.42-1.31)$ & 0.30 & 2.79 (95\% CI 0.82-9.46) & 0.10 \\
\hline Tumor site & $1.24(95 \%$ CI $0.76-2.04)$ & 0.39 & 1.35 (95\% CI 0.81-2.25) & 0.24 & $1.40(95 \%$ CI $0.62-3.15)$ & 0.41 \\
\hline T-Classification & 1.17 (95\% CI 0.43-3.13) & 0.76 & $0.75(95 \%$ CI $0.22-2.58)$ & 0.65 & 1.44 (95\% CI 0.29-7.12) & 0.66 \\
\hline N-Classification & $1.14(95 \%$ CI $0.38-3.43)$ & 0.82 & 5.84 (95\% CI 0.88-38.9) & 0.07 & 2.28 (95\% CI $0.21-25.1)$ & 0.50 \\
\hline Total tumor volume & $2.46(95 \%$ CI $1.47-4.11)$ & $<0.01$ & $2.50(95 \%$ CI $1.43-4.36)$ & $<0.01$ & $1.59(95 \%$ CI $0.78-3.26)$ & 0.20 \\
\hline $\begin{array}{l}\text { Simultaneous } \\
\text { radiochemotherapy }\end{array}$ & $0.52(95 \%$ CI $0.31-0.88)$ & 0.01 & 0.68 (95\% CI $0.40-1.16)$ & 0.15 & $2.63(95 \%$ CI $1.03-6.73)$ & 0.04 \\
\hline RT interruptions $>5$ days & 0.95 (95\% CI $0.5-1.8)$ & 0.88 & 0.77 (95\% CI $0.36-1.66)$ & 0.51 & 1.08 (95\% CI $0.39-3.04)$ & 0.88 \\
\hline
\end{tabular}

RT, radiation therapy; RR, relative risk, $P$ : $P$-value. 
TABLE 4: Postoperative radiation therapy: univariate analysis of overall survival, locoregional control, and metastasis-free survival.

\begin{tabular}{|c|c|c|c|c|c|c|}
\hline \multirow{2}{*}{ Factor } & \multicolumn{2}{|l|}{ Overall survival } & \multicolumn{2}{|l|}{ Locoregional failure } & \multicolumn{2}{|l|}{ Metastasis } \\
\hline & $5 y$-survival & $P$ & $5 y-C I F$ & $P$ & $5 y-C I F$ & $P$ \\
\hline \multicolumn{7}{|l|}{ Gender } \\
\hline Female & 0.89 (95\% CI 0.75-1.03) & & $0.53(95 \%$ CI $0.10-0.85)$ & & 0.11 (95\% CI 0.02-0.29) & \\
\hline Male & 0.66 (95\% CI 0.54-0.77) & 0.17 & $0.33(95 \%$ CI $0.23-0.44)$ & 0.56 & $0.21(95 \%$ CI $0.14-0.30)$ & 0.27 \\
\hline \multicolumn{7}{|l|}{ Age (years) } \\
\hline$\leq 56$ & $0.72(95 \%$ CI $0.60-0.84)$ & & $0.29(95 \%$ CI $0.17-0.41)$ & & $0.21(95 \%$ CI $0.12-0.31)$ & \\
\hline$>56$ & 0.65 (95\% CI 0.47-0.82) & 0.87 & $0.45(95 \%$ CI $0.27-0.61)$ & 0.10 & 0.19 (95\% CI 0.09-0.31) & 0.85 \\
\hline \multicolumn{7}{|c|}{$\begin{array}{l}\text { Hemoglobin level pre-RT } \\
(\mathrm{g} / \mathrm{dL})\end{array}$} \\
\hline$\leq 12$ & 0.64 (95\% CI 0.46-0.83) & & 0.41 (95\% CI 0.20-0.61) & & 0.17 (95\% CI 0.07-0.31) & \\
\hline$>12$ & 0.69 (95\% CI 0.55-0.84) & 0.72 & 0.27 (95\% CI 0.16-0.40) & 0.42 & 0.23 (95\% CI 0.13-0.35) & 0.45 \\
\hline \multicolumn{7}{|l|}{ Tumor site } \\
\hline Oropharynx & $0.72(95 \%$ CI $0.58-0.86)$ & & 0.44 (95\% CI 0.27-0.59) & & 0.14 (95\% CI 0.07-0.23) & \\
\hline Hypopharynx & 0.65 (95\% CI 0.51-0.80) & 0.35 & $0.28(95 \%$ CI $0.16-0.41)$ & 0.46 & 0.27 (95\% CI $0.15-0.40)$ & 0.30 \\
\hline \multicolumn{7}{|l|}{ T-Classification } \\
\hline 1,2 & 0.69 (95\% CI 0.54-0.83) & & 0.34 (95\% CI 0.20-0.49) & & $0.19(95 \%$ CI $0.11-0.30)$ & \\
\hline 3,4 & 0.70 (95\% CI 0.56-0.83) & 0.80 & 0.37 (95\% CI 0.23-0.52) & 0.60 & 0.20 (95\% CI 0.11-0.32) & 0.94 \\
\hline \multicolumn{7}{|l|}{ N-Classification } \\
\hline 0,1 & $0.72(95 \%$ CI $0.54-0.89)$ & & 0.28 (95\% CI 0.12-0.48) & & $0.10(95 \%$ CI $0.04-0.21)$ & \\
\hline 2,3 & $0.68(95 \%$ CI $0.55-0.80)$ & 0.24 & $0.40(95 \%$ CI $0.27-0.53)$ & 0.06 & $0.26(95 \%$ CI $0.16-0.36)$ & 0.03 \\
\hline \multicolumn{7}{|l|}{ R-Classification } \\
\hline 0 & $0.68(95 \%$ CI $0.56-0.80)$ & & 0.37 (95\% CI 0.23-0.50) & & $0.21(95 \%$ CI $0.11-0.31)$ & \\
\hline 1,2 & 0.69 (95\% CI 0.59-0.79) & 0.95 & 0.37 (95\% CI 0.16-0.58) & 0.81 & 0.20 (95\% CI 0.07-0.33) & 0.80 \\
\hline \multicolumn{7}{|c|}{ Total tumor volume $\left(\mathrm{cm}^{3}\right)$} \\
\hline$\leq 21.2$ & 0.65 (95\% CI 0.47-0.82) & & 0.23 (95\% CI 0.09-0.37) & & 0.17 (95\% CI 0.07-0.27) & \\
\hline$>21.2$ & $0.61(95 \%$ CI $0.45-0.76)$ & 0.13 & 0.44 (95\% CI 0.27-0.60) & 0.04 & $0.26(95 \%$ CI $0.14-0.39)$ & 0.25 \\
\hline \multicolumn{7}{|c|}{ RT interruptions $>5$ days } \\
\hline Yes & 0.66 (95\% CI 0.45-0.87) & & 0.51 (95\% CI 0.26-0.72) & & 0.25 (95\% CI $0.10-0.44)$ & \\
\hline No & 0.70 (95\% CI 0.57-0.82) & 0.22 & 0.27 (95\% CI 0.17-0.39) & 0.10 & 0.18 (95\% CI $0.11-0.27)$ & 0.26 \\
\hline \multicolumn{7}{|c|}{ Interval surgery-RT $>32$ days } \\
\hline Yes & 0.66 (95\% CI 0.51-0.80) & 0.50 & $0.45(95 \%$ CI $0.28-0.60)$ & 0.40 & 0.20 (95\% CI 0.11-0.32) & 0.79 \\
\hline No & $0.70(95 \%$ CI $0.54-0.85)$ & & $0.27(95 \%$ CI $0.15-0.40)$ & & $0.20(95 \%$ CI $0.11-0.32)$ & \\
\hline
\end{tabular}

RT: radiation therapy; CIF: cumulative incidence function; $P: P$ value.

radiotherapy/radiochemotherapy or (ii) surgery followed by postoperative radiotherapy. The two patient groups were analysed separately because they differed considerably in respect to patient- and treatment-related characteristics and prognosis.

The tumor volume has been stated to be one of the most precise and most relevant predictor of radiotherapy outcome [9]. For patients with oro- and hypopharyngeal cancer treated with definitive radiotherapy or radiochemotherapy, the quantitative tumor volume was identified as significant prognostic factor in the majority of studies [10-20], and in few studies, as a prognostic factor of marginal $[21,22]$ or no significance [23].

In the multivariate analysis of our study, the total tumor volume had a statistically highly significant impact on the overall survival and locoregional control on patients treated with definitive radiotherapy/radiochemotherapy.
A new finding of our study is that the total tumor volume also had a statistically significant impact on the locoregional control in patients treated with surgery followed by postoperative radiotherapy. For this patient group, the total tumor volume was the only significant prognostic factor in the multivariate analysis. Our data suggest that the total tumor volume should be used to select patients for an intensified definitive or postoperative adjuvant treatment. Recent randomized studies have shown a significantly improved outcome of "high-risk" patients treated with adjuvant simultaneous radiochemotherapy compared to adjuvant radiotherapy alone [24, 25]. A comparative analysis of both studies revealed the extracapsular extension of tumor from neck nodes and/or microscopically involved surgical margins as significant clinical risk factors for poor outcome [26]. The quantitative total tumor volume was not considered in this analysis. 
TABLE 5: Postoperative radiation therapy: multivariate analysis of overall survival, locoregional control, and metastasis-free survival.

\begin{tabular}{|c|c|c|c|c|c|c|}
\hline \multirow{2}{*}{ Factor } & \multicolumn{2}{|c|}{ Overall survival } & \multicolumn{2}{|c|}{ Locoregional control } & \multicolumn{2}{|c|}{ Metastasis-free survival } \\
\hline & $\mathrm{RR}$ & $P$ & $\mathrm{RR}$ & $P$ & $\mathrm{RR}$ & $P$ \\
\hline Gender & $\begin{array}{c}0.34(95 \% \mathrm{CI} \\
0.04-2.74)\end{array}$ & 0.31 & $\begin{array}{c}0.33(95 \% \text { CI } \\
0.05-2.10)\end{array}$ & 0.24 & $\begin{array}{c}0.63(95 \% \text { CI } \\
0.05-8.47)\end{array}$ & 0.73 \\
\hline Age & $\begin{array}{c}1.11(95 \% \text { CI } \\
0.49-2.54)\end{array}$ & 0.80 & $\begin{array}{c}2.23(95 \% \mathrm{CI} \\
0.93-5.38)\end{array}$ & 0.07 & $\begin{array}{c}1.05(95 \% \mathrm{CI} \\
0.36-3.04)\end{array}$ & 0.93 \\
\hline Hemoglobin level pre-RT & $\begin{array}{c}0.88(95 \% \text { CI } \\
0.40-1.92)\end{array}$ & 0.75 & $\begin{array}{c}0.79(95 \% \text { CI } \\
0.32-1.93)\end{array}$ & 0.60 & $\begin{array}{c}1.42(95 \% \text { CI } \\
0.55-3.68)\end{array}$ & 0.47 \\
\hline Tumor site & $\begin{array}{c}0.98(95 \% \mathrm{CI} \\
0.42-2.26)\end{array}$ & 0.95 & $\begin{array}{c}1.25(95 \% \mathrm{CI} \\
0.46-3.42)\end{array}$ & 0.66 & $\begin{array}{c}0.65(95 \% \text { CI } \\
0.25-1.72)\end{array}$ & 0.39 \\
\hline T-Classification & $\begin{array}{c}1.24(95 \% \text { CI } \\
0.53-2.88)\end{array}$ & 0.62 & $\begin{array}{c}1.85(95 \% \text { CI } \\
0.77-4.42)\end{array}$ & 0.17 & $\begin{array}{c}1.24(95 \% \mathrm{CI} \\
0.47-3.26)\end{array}$ & 0.66 \\
\hline N-Classification & $\begin{array}{c}1.17(95 \% \text { CI } \\
0.45-3.09)\end{array}$ & 0.75 & $\begin{array}{c}1.81(95 \% \text { CI } \\
0.61-5.35)\end{array}$ & 0.28 & $\begin{array}{c}2.25(95 \% \mathrm{CI} \\
0.72-7.01)\end{array}$ & 0.16 \\
\hline R-Classification & $\begin{array}{c}1.17(95 \% \mathrm{CI} \\
0.50-2.73)\end{array}$ & 0.72 & $\begin{array}{c}0.79(95 \% \text { CI } \\
0.34-1.87)\end{array}$ & 0.60 & $\begin{array}{c}1.04(95 \% \text { CI } \\
0.41-2.64)\end{array}$ & 0.94 \\
\hline Total tumor volume & $\begin{array}{c}1.77(95 \% \text { CI } \\
0.76-4.14)\end{array}$ & 0.19 & $\begin{array}{c}3.19(95 \% \text { CI } \\
1.00-10.2)\end{array}$ & 0.05 & $\begin{array}{c}1.03(95 \% \mathrm{CI} \\
0.37-2.85)\end{array}$ & 0.95 \\
\hline RT interruptions $>5$ days & $\begin{array}{c}1.42(95 \% \mathrm{CI} \\
0.57-3.50)\end{array}$ & 0.45 & $\begin{array}{c}1.17(95 \% \mathrm{CI} \\
0.50-2.70)\end{array}$ & 0.72 & $\begin{array}{c}1.15(95 \% \mathrm{CI} \\
0.31-4.31)\end{array}$ & 0.84 \\
\hline Interval surgery-RT >32 days & $\begin{array}{c}1.45(95 \% \mathrm{CI} \\
0.67-3.13)\end{array}$ & 0.35 & $\begin{array}{c}1.92(95 \% \mathrm{CI} \\
0.81-4.54)\end{array}$ & 0.14 & $\begin{array}{c}0.96(95 \% \mathrm{CI} \\
0.38-2.41)\end{array}$ & 0.93 \\
\hline
\end{tabular}

RT: radiation therapy; RR: relative risk; $P: P$ value.

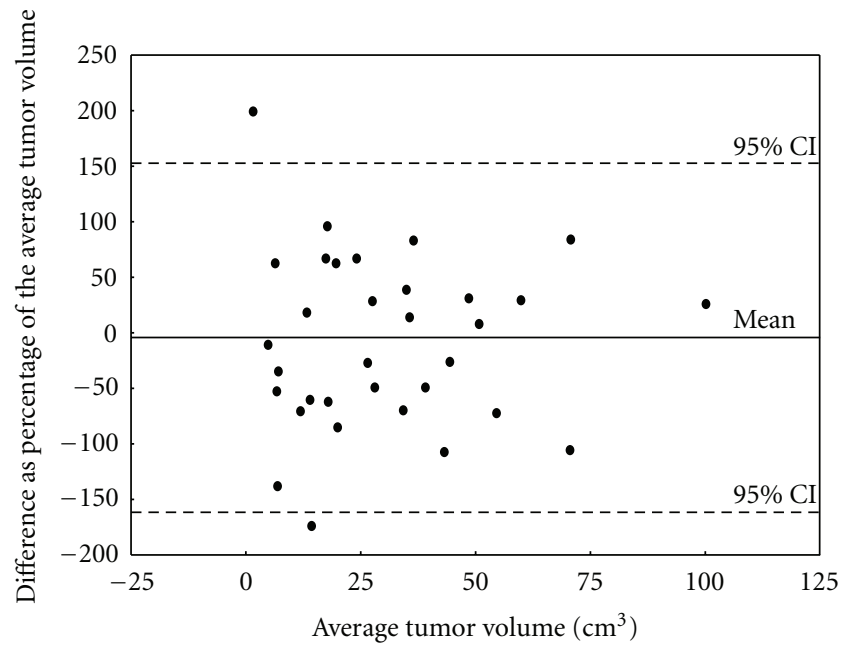

FIGURE 4: Difference as percentage of the average tumor volume of the quantitative CT-based tumor volumetry and estimation of the tumor volume based on the histological report plotted against their mean.

The only other significant prognostic factor in the multivariate analysis for patients treated with definitive radiotherapy in our study was the application of a simultaneous chemotherapy. The simultaneous chemotherapy was significantly associated with an improved overall survival and distant metastasis-free survival and showed a statistical trend of an improved locoregional control $(P=0.15)$. This observation is well in line with the literature. Randomized clinical trials [27-35] and meta-analyses [36, 37] have shown a significantly improved local control and survival with definitive simultaneous radiochemotherapy compared to definitive radiotherapy alone in patients with advanced squamous cell cancer of the head and neck.

Interestingly, in our study, the preradiotherapy hemoglobin level was a significant prognostic factor in the univariate analysis of patients treated with definitive radiotherapy/radiochemotherapy but lost its statistical significance in the multivariate analysis. If the total tumor volume was removed from the multivariate model, the pre-radiotherapy hemoglobin level retained its significance. Our data suggest that the total tumor volume is the stronger of both prognostic factors. A significant association of the pre-radiotherapy hemoglobin concentration with the treatment outcome after definitive radiotherapy/radiochemotherapy in the absence of quantitative tumor volume data has been reported by several studies in the literature [38-46].

For patients treated with postoperative radiotherapy, the pre-radiotherapy hemoglobin level showed no prognostic significance in our study. In the literature, differing findings are reported. One study found a significant impact of the preradiotherapy hemoglobin level on survival in patients with head and neck cancer treated with adjuvant radiotherapy [47]. Other studies evaluated the prognostic significance of the hemoglobin level at different time points during the treatment. In these studies, no prognostic significance was found for the pre-radiotherapy hemoglobin level, but for the hemoglobin level before surgery [48], after surgery $[49,50]$, duration of low hemoglobin level during the interval between surgery and radiotherapy [48], or for the difference of the hemoglobin concentration before and after adjuvant radiotherapy [51]. Several studies using $\mathrm{pO}_{2}$ 
histography have shown an impact of the tumor oxygenation on the survival of patients with head and neck cancer after radiotherapy [52-55], but no clear correlation was found between the tumor oxygenation by means of $\mathrm{pO}_{2}$ histography and the hemoglobin concentration $[56,57]$.

On multivariate analysis, the potential prognostic factors, gender, age, pre-radiotherapy hemoglobin level, tumor site, $\mathrm{T}$ - and N-, and R-status, RT-interruptions $>5$ days, and interval surgery-RT $>32$ days, showed no statistical significance in our study.

Other prognostic factors on multivariate analysis for locally advanced head and neck cancers reported by other studies were the performance status [45, 46], high-grade acute organ toxicity [58], or the UICC stage [59].

\section{Conclusion}

Our data suggest that the total tumor volume is the predominant prognostic factor in patients with squamous cell cancer of the oro- and hypopharynx treated with definitive radiotherapy/radiochemotherapy or surgery followed by postoperative radiotherapy. The total tumor volume should be used to identify high-risk patients and to stratify patients in clinical trials or statistical analyses.

\section{References}

[1] D. M. Parkin, F. Bray, J. Ferlay, and P. Pisani, "Estimating the world cancer burden: globocan 2000," International Journal of Cancer, vol. 94, no. 2, pp. 153-156, 2001.

[2] S. G. Laskar, J. P. Agarwal, C. Srinivas, and K. A. Dinshaw, "Radiotherapeutic management of locally advanced head and neck cancer," Expert Review of Anticancer Therapy, vol. 6, no. 3, pp. 405-417, 2006.

[3] R. Corvò, "Evidence-based radiation oncology in head and neck squamous cell carcinoma," Radiotherapy and Oncology, vol. 85, no. 1, pp. 156-170, 2007.

[4] V. Rudat and M. Wannenmacher, "Role of multimodal treatment in oropharynx, larynx, and hypopharynx cancer," Seminars in Surgical Oncology, vol. 20, no. 1, pp. 66-74, 2001.

[5] R. Gray, "A class of K-sample tests for comparing the cumulative incidence of a competing risk," Annals of Statistics, vol. 16, pp. 1141-1154, 1988.

[6] N. Mantel, "Evaluation of survival data and two new rank order statistics arising in its consideration," Cancer Chemotherapy Reports, vol. 50, no. 3, pp. 163-170, 1966.

[7] J. P. Fine and R. J. Gray, "A proportional hazards model for the subdistribution of a competing risk," Journal of the American Statistical Association, vol. 94, no. 446, pp. 496-509, 1999.

[8] J. M. Bland and D. G. Altman, "Statistical methods for assessing agreement between two methods of clinical measurement," The Lancet, vol. 1, no. 8476, pp. 307-310, 1986.

[9] H. H. Dubben, H. D. Thames, and H. P. Beck-Bornholdt, "Tumor volume: a basic and specific response predictor in radiotherapy," Radiotherapy and Oncology, vol. 47, no. 2, pp. 167-174, 1998.

[10] F. J. P. Hoebers, F. A. Pameijer, J. de Bois et al., "Prognostic value of primary tumor volume after concurrent chemoradiation with daily low-dose cisplatin for advanced-stage head and neck carcinoma," Head and Neck, vol. 30, no. 9, pp. 1216-1223, 2008.
[11] A. T. Yung, H. H. Jun, H. L. Meng, and H. T. Ming, "Analysis of prognostic factors of chemoradiation therapy for advanced hypopharyngeal cancer-does tumor volume correlate with central necrosis and tumor pathology?" Oto Rhino Laryngology, vol. 68, no. 4, pp. 206-212, 2006.

[12] S. W. Chen, S. N. Yang, J. A. Liang, M. H. Tsai, A. C. Shiau, and F. J. Lin, "Value of computed tomography-based tumor volume as a predictor of outcomes in hypopharyngeal cancer after treatment with definitive radiotherapy," Laryngoscope, vol. 116, no. 11, pp. 2012-2017, 2006.

[13] G. A. Plataniotis, M. E. Theofanopoulou, A. KalogeraFountzila et al., "Prognostic impact of tumor volumetry in patients with locally advanced head-and-neck carcinoma (non-nasopharyngeal) treated by radiotherapy alone or combined radiochemotherapy in a randomized trial," International Journal of Radiation Oncology Biology Physics, vol. 59, no. 4, pp. 1018-1026, 2004.

[14] K. S. C. Chao, G. Ozyigit, A. I. Blanco et al., "Intensitymodulated radiation therapy for oropharyngeal carcinoma: impact of tumor volume," International Journal of Radiation Oncology Biology Physics, vol. 59, no. 1, pp. 43-50, 2004.

[15] I. Doweck, D. Denys, and K. T. Robbins, "Tumor volume predicts outcome for advanced head and neck cancer treated with targeted chemoradiotherapy," Laryngoscope, vol. 112, no. 10, pp. 1742-1749, 2002.

[16] G. G. Grabenbauer, H. Steininger, M. Meyer et al., "Nodal CT density and total tumor volume as prognostic factors after radiation therapy of stage III/IV head and neck cancer," Radiotherapy and Oncology, vol. 47, no. 2, pp. 175-183, 1998.

[17] C. R. Johnson, S. R. Khandelwal, R. K. Schmidt-Ullrich, J. Ravalese III, and D. E. Wazer, "The influence of quantitative tumor volume measurements on local control in advanced head and neck cancer using concomitant boost accelerated superfractionated irradiation," International Journal of Radiation Oncology Biology Physics, vol. 32, no. 3, pp. 635-641, 1995.

[18] V. Rudat, A. Dietz, O. Schramm et al., "Prognostic impact of total tumor volume and hemoglobin concentration on the outcome of patients with advanced head and neck cancer after concomitant boost radiochemotherapy," Radiotherapy and Oncology, vol. 53, no. 2, pp. 119-125, 1999.

[19] V. Rudat, H. Eckel, P. Volling et al., "Long-term results of a prospective multicenter phase II study to preserve the larynx function using concomitant boost radiochemotherapy with carboplatin," Radiotherapy and Oncology, vol. 89, no. 1, pp. 33-37, 2008.

[20] R. Kurek, A. Kalogera-Fountzila, K. Muskalla et al., "Usefulness of tumor volumetry as a prognostic factor of survival in head and neck cancer," Strahlentherapie und Onkologie, vol. 179, no. 5, pp. 292-297, 2003.

[21] R. Hermans, K. op de beeck, W. van den Bogaert et al., "The relation of CT-determined tumor parameters and local and regional outcome of tonsillar cancer after definitive radiation treatment," International Journal of Radiation Oncology Biology Physics, vol. 50, no. 1, pp. 37-45, 2001.

[22] R. M. Nathu, A. A. Mancuso, T. C. Zhu, and W. M. Mendenhall, "The impact of primary tumor volume on local control for oropharyngeal squamous cell carcinoma treated with radiotherapy," Head and Neck, vol. 22 , no. 1, pp. 1-5, 2000.

[23] M. J. Been, J. Watkins, R. M. Manz et al., "Tumor volume as a prognostic factor in oropharyngeal squamous cell carcinoma treated with primary radiotherapy," Laryngoscope, vol. 118, no. 8, pp. 1377-1382, 2008. 
[24] J. Bernier, C. Domenge, M. Ozsahin et al., "Postoperative irradiation with or without concomitant chemotherapy for locally advanced head and neck cancer," The New England Journal of Medicine, vol. 350, no. 19, pp. 1945-1952, 2004.

[25] J. S. Cooper, T. F. Pajak, A. A. Forastiere et al., "Postoperative concurrent radiotherapy and chemotherapy for high-risk squamous-cell carcinoma of the head and neck," The New England Journal of Medicine, vol. 350, no. 19, pp. 1937-1944, 2004.

[26] J. Bernier, J. S. Cooper, T. F. Pajak et al., "Defining risk levels in locally advanced head and neck cancers: a comparative analysis of concurrent postoperative radiation plus chemotherapy trials of the EORTC (\#22931) and RTOG (\#9501)," Head and Neck, vol. 27, no. 10, pp. 843-850, 2005.

[27] T. G. Wendt, G. G. Grabenbauer, C. M. Rödel et al., "Simultaneous radiochemotherapy versus radiotherapy alone in advanced head and neck cancer: a randomized multicenter study," Journal of Clinical Oncology, vol. 16, no. 4, pp. 13181324, 1998.

[28] D. M. Brizel, M. E. Albers, S. R. Fisher et al., "Hyperfractionated irradiation with or without concurrent chemotherapy for locally advanced head and neck cancer," The New England Journal of Medicine, vol. 338, no. 25, pp. 1798-1804, 1998.

[29] G. Calais, M. Alfonsi, E. Bardet et al., "Randomized trial of radiation therapy versus concomitant chemotherapy and radiation therapy for advanced-stage oropharynx carcinoma," Journal of the National Cancer Institute, vol. 91, no. 24, pp. 2081-2086, 1999.

[30] V. Budach, M. Stuschke, W. Budach et al., "Hyperfractionated accelerated chemoradiation with concurrent fluorouracilmitomycin is more effective than dose-escalated hyperfractionated accelerated radiation therapy alone in locally advanced head and neck cancer: final results of the radiotherapy cooperative clinical trials group of the German Cancer Society 95-06 prospective randomized trial," Journal of Clinical Oncology, vol. 23, no. 6, pp. 1125-1135, 2005.

[31] S. Staar, V. Rudat, H. Stuetzer et al., "Intensified hyperfractionated accelerated radiotherapy limits the additional benefit of simultaneous chemotherapy-results of a multicentric randomized German trial in advanced head-and-neck cancer," International Journal of Radiation Oncology Biology Physics, vol. 50, no. 5, pp. 1161-1171, 2001.

[32] P. Huguenin, K. T. Beer, A. Allal et al., "Concomitant cisplatin significantly improves locoregional control in advanced head and neck cancers treated with hyperfractionated radiotherapy," Journal of Clinical Oncology, vol. 22, no. 23, pp. 46654673, 2004.

[33] D. J. Adelstein, Y. Li, G. L. Adams et al., "An intergroup phase III comparison of standard radiation therapy and two schedules of concurrent chemoradiotherapy in patients with unresectable squamous cell head and neck cancer," Journal of Clinical Oncology, vol. 21, no. 1, pp. 92-98, 2003.

[34] F. Denis, P. Garaud, E. Bardet et al., "Final results of the 9401 French head and neck oncology and radiotherapy group randomized trial comparing radiotherapy alone with concomitant radiochemotherapy in advanced-stage oropharynx carcinoma," Journal of Clinical Oncology, vol. 22, no. 1, pp. 6976, 2004.

[35] B. Jeremic, Y. Shibamoto, B. Milicic et al., "Hyperfractionated radiation therapy with or without concurrent low-dose daily cisplatin in locally advanced squamous cell carcinoma of the head and neck: a prospective randomized trial," Journal of Clinical Oncology, vol. 18, no. 7, pp. 1458-1464, 2000.
[36] J. P. Pignon, A. 1. Maître, E. Maillard, and J. Bourhis, "Metaanalysis of chemotherapy in head and neck cancer (MACHNC): an update on 93 randomised trials and 17,346 patients," Radiotherapy and Oncology, vol. 92, no. 1, pp. 4-14, 2009.

[37] J. Bourhis, J. Overgaard, H. Audry et al., "Hyperfractionated or accelerated radiotherapy in head and neck cancer: a metaanalysis," The Lancet, vol. 368, no. 9538, pp. 843-854, 2006.

[38] A. Fortin, C. S. Wang, and É. Vigneault, "Effect of pretreatment anemia on treatment outcome of concurrent radiochemotherapy in patients with head and neck cancer," International Journal of Radiation Oncology Biology Physics, vol. 72, no. 1, pp. 255-260, 2008.

[39] D. Rades, M. Lange, T. Veninga et al., "Preliminary results of spinal cord compression recurrence evaluation (score-1) study comparing short-course versus long-course radiotherapy for local control of malignant epidural spinal cord compression," International Journal of Radiation Oncology Biology Physics, vol. 73, no. 1, pp. 228-234, 2009.

[40] R. G. Prosnitz, B. Yao, C. L. Farrell, R. Clough, and D. M. Brizel, "Pretreatment anemia is correlated with the reduced effectiveness of radiation and concurrent chemotherapy in advanced head and neck cancer," International Journal of Radiation Oncology Biology Physics, vol. 61, no. 4, pp. 10871095, 2005.

[41] U. Schäfer, O. Micke, S. B. Müller, P. Schüller, and N. Willich, "Hemoglobin as an independent prognostic factor in the radiotherapy of head and neck tumors," Strahlentherapie und Onkologie, vol. 179, no. 8, pp. 527-534, 2003.

[42] W. R. Lee, B. Berkey, V. Marcial et al., "Anemia is associated with decreased survival and increased locoregional failure in patients with locally advanced head and neck carcinoma: a secondary analysis of RTOG 85-27," International Journal of Radiation Oncology Biology Physics, vol. 42, no. 5, pp. 10691075, 1998.

[43] B. Dubray, V. Mosseri, F. Brunin et al., "Anemia is associated with lower local-regional control and survival after radiation therapy for head and neck cancer: a prospective study," Radiology, vol. 201, no. 2, pp. 553-558, 1996.

[44] R. Semrau, R. P. Mueller, H. Stuetzer et al., "Efficacy of intensified hyperfractionated and accelerated radiotherapy and concurrent chemotherapy with carboplatin and 5fluorouracil: updated results of a randomized multicentric trial in advanced head-and-neck cancer," International Journal of Radiation Oncology Biology Physics, vol. 64, no. 5, pp. 13081316, 2006.

[45] D. Rades, N. D. Seibold, M. P. Gebhard, F Noack, S. E. Schild, and C. Thorns, "Prognostic factors (including HPV status) for irradiation of locally advanced squamous cell carcinoma of the head and neck (SCCHN)," Strahlentherapie und Onkologie, vol. 187, no. 10, pp. 626-632, 2011.

[46] D. Rades, T. Meyners, N. Kazic, A. Bajrovic, V. Rudat, and S. E. Schild, "Comparison of radiochemotherapy alone to surgery plus radio(chemo)therapy for non-metastatic stage III/IV squamous cell carcinoma of the head and neck: a matched-pair analysis," Strahlentherapie und Onkologie, vol. 187, no. 9, pp. 541-547, 2011.

[47] A. G. Macdonald and D. C. Hurman, "Influence of anaemia in patients with head and neck cancer receiving adjuvant postoperative radiotherapy in the Grampian region," Clinical Oncology, vol. 16, no. 1, pp. 63-70, 2004.

[48] S. M. G. van de Pol, P. A. H. Doornaert, R. de Bree, C. R. Leemans, B. J. Slotman, and J. A. Langendijk, "The significance of anemia in squamous cell head and neck cancer treated with 
surgery and postoperative radiotherapy," Oral Oncology, vol. 42, no. 2, pp. 131-138, 2006.

[49] B. Dietl, J. Marienhagen, C. Schäfer, and O. Kölbl, "The prognostic value of anaemia at different treatment times in patients with locally advanced head and neck cancer treated with surgery and postoperative radiotherapy," Clinical Oncology, vol. 19, no. 4, pp. 228-233, 2007.

[50] O. Reichel, M. Panzer, C. Wimmer, E. Dühmke, E. Kastenbauer, and M. Suckfüll, "Prognostic implications of hemoglobin levels before and after surgery as well as before and after radiochemotherapy for head and neck tumors," European Archives of Oto-Rhino-Laryngology, vol. 260, no. 5, pp. 248-253, 2003.

[51] T. Rutkowski, R. Suwinski, and A. Idasiak, "The prognostic value of hemoglobin concentration in postoperative radiotherapy of 835 patients with laryngeal cancer," International Journal of Radiation Oncology Biology Physics, vol. 69, no. 4, pp. 1018-1023, 2007.

[52] M. Nordsmark, S. M. Bentzen, V. Rudat et al., "Prognostic value of tumor oxygenation in 397 head and neck tumors after primary radiation therapy. An international multi-center study," Radiotherapy and Oncology, vol. 77, no. 1, pp. 18-24, 2005.

[53] V. Rudat, P. Stadler, A. Becker et al., "Predictive value of the tumor oxygenation by means of $\mathrm{pO} 2$ histography in patients with advanced head and neck cancer," Strahlentherapie und Onkologie, vol. 177, no. 9, pp. 462-468, 2001.

[54] A. Becker, G. Hänsgen, M. Blocking, C. Weigel, C. Lautenschläger, and J. Dunst, "Oxygenation of squamous cell carcinoma of the head and neck: comparison of primary tumors, neck node metastases, and normal tissue," International Journal of Radiation Oncology Biology Physics, vol. 42, no. 1, pp. 35-41, 1998.

[55] P. Stadler, A. Becker, H. Jürgen Feldmann et al., "Influence of the hypoxic subvolume on the survival of patients with head and neck cancer," International Journal of Radiation Oncology Biology Physics, vol. 44, no. 4, pp. 749-754, 1999.

[56] V. Rudat, B. Vanselow, P. Wollensack et al., "Repeatability and prognostic impact of the pretreatment pO2 histography in patients with advanced head and neck cancer," Radiotherapy and Oncology, vol. 57, no. 1, pp. 31-37, 2000.

[57] M. Nordsmark and J. Overgaard, "Tumor hypoxia is independent of hemoglobin and prognostic for loco-regional tumor control after primary radiotherapy in advanced head and neck cancer," Acta Oncologica, vol. 43, no. 4, pp. 396-403, 2004.

[58] H. A. Wolff, J. Bosch, K. Jung et al., "High-grade acute organ toxicity as positive prognostic factor in primary radio(chemo)therapy for locally advanced, inoperable head and neck cancer," Strahlentherapie und Onkologie, vol. 186, no. 5, pp. 262-268, 2010.

[59] M. Kreppel, U. Drebber, H. T. Eich et al., "Combined-modality treatment in advanced oral squamous cell carcinoma: primary surgery followed by adjuvant concomitant radiochemotherapy," Strahlentherapie und Onkologie, vol. 187, no. 9, pp. 555560,2011 


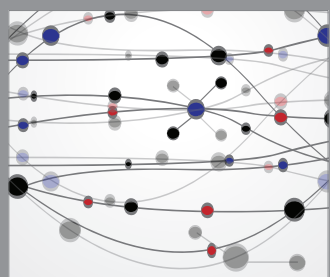

The Scientific World Journal
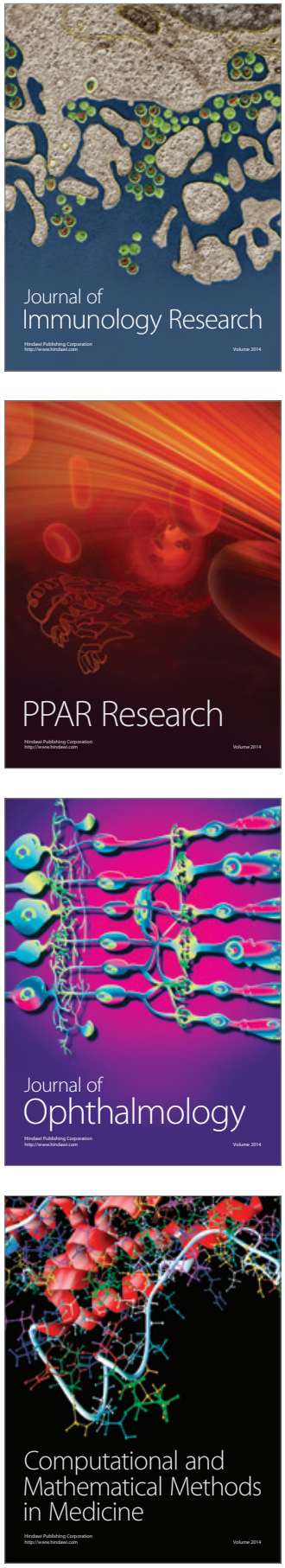

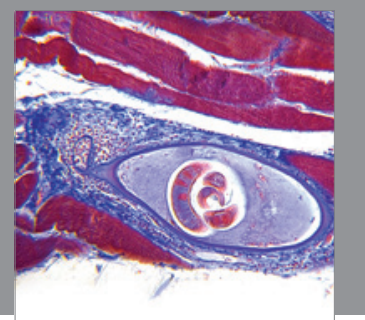

Gastroenterology

Research and Practice
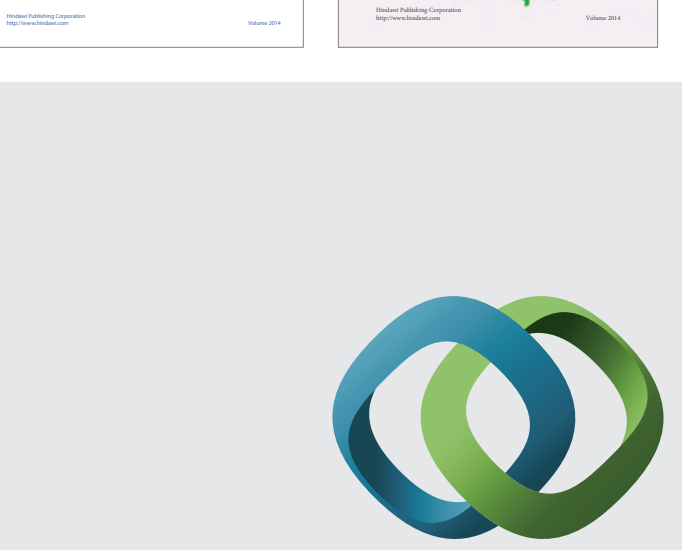

\section{Hindawi}

Submit your manuscripts at

http://www.hindawi.com
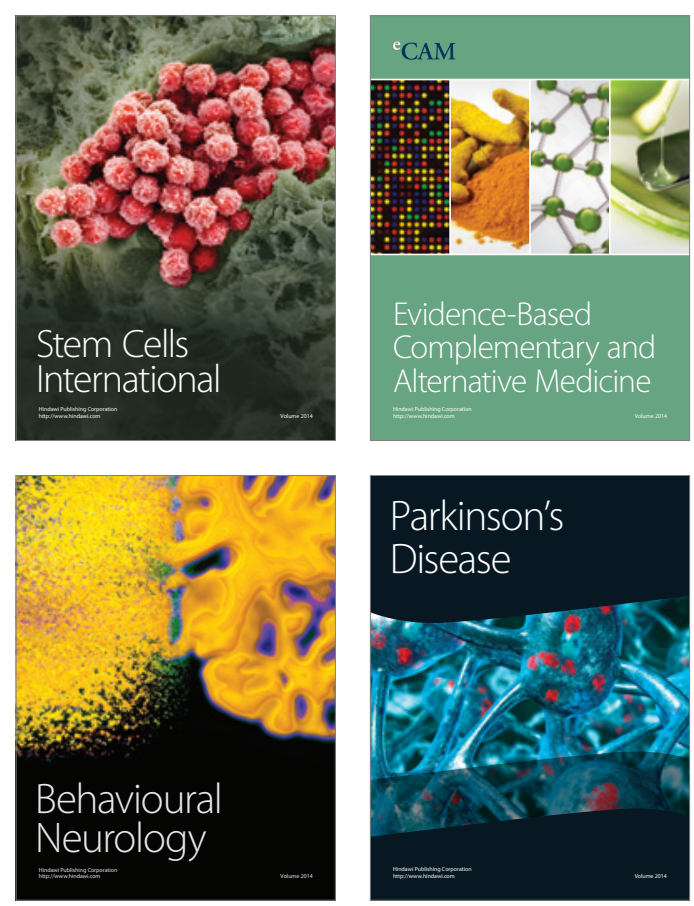

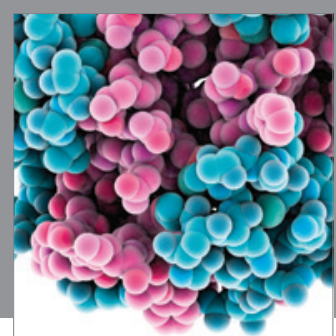

Journal of
Diabetes Research

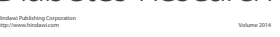

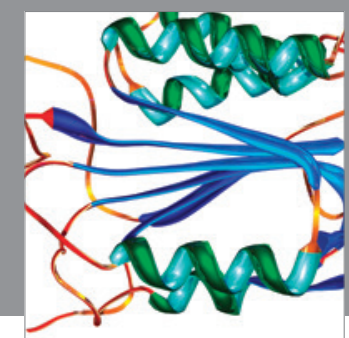

Disease Markers
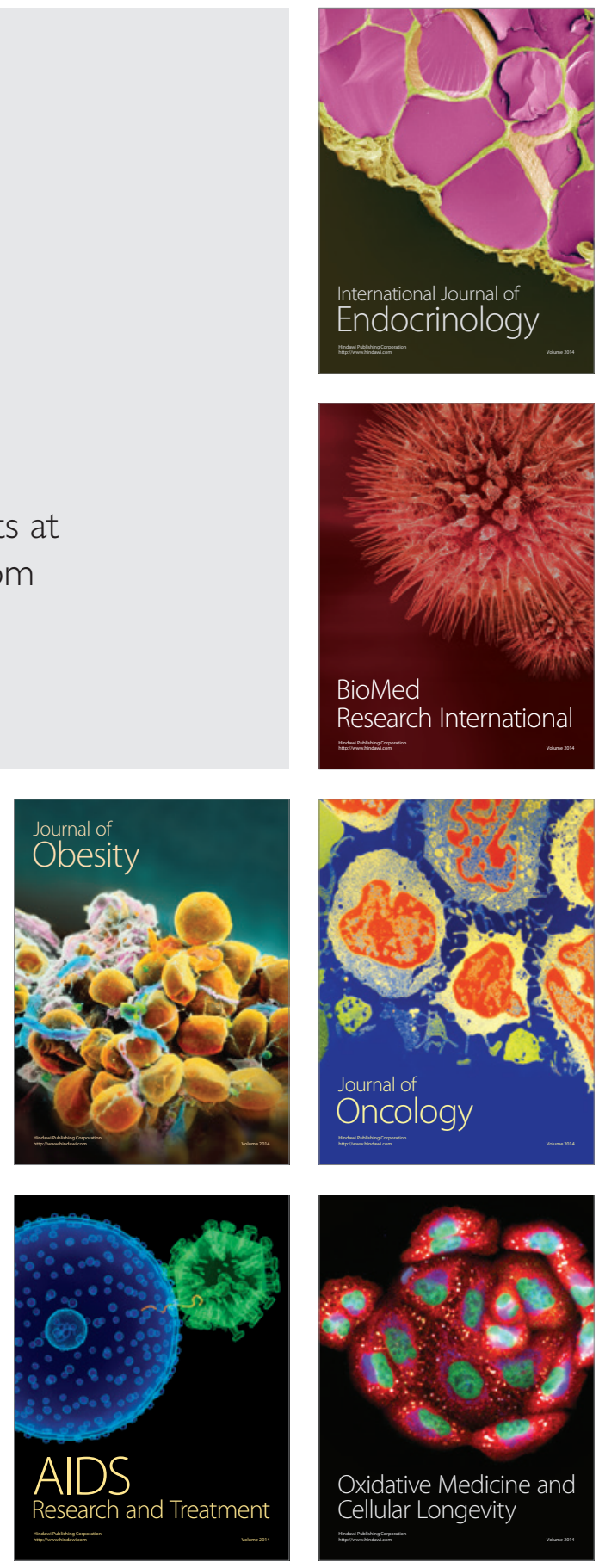